\title{
Successful operation in an old survivor of anomalous origin of the left coronary artery from the pulmonary trunk (Bland-White-Garland syndrome)
}

\author{
Basil T Saeed, Michael D Rosin, R Gordon Murray
}

\begin{abstract}
A case of anomalous origin of the left coronary artery from the pulmonary trunk is reported. The patient, a 64-yearold woman, presented with a history of angina and cardiac failure. She was known to have had a mitral systolic murmur since school age. Echocardiography showed clinically significant mitral regurgitation and highly unusual extensive calcification of the mitral valve chordae, papillary muscle, and posterior left ventricular wall-a pattern suggesting the possibility of abnormal coronary circulation. Subsequent cardiac catheterisation confirmed considerable mitral regurgitation with a dilated left ventricle, and arteriography confirmed anomalous origin of the left coronary artery from the main pulmonary trunk. The patient was surgically treated with ligation of the origin of the anomalous left coronary and mitral valve replacement. She was alive and well 2 years after operation.
\end{abstract}

(Br Heart f 1994;71:193-195)

Origin of the left coronary artery from the pulmonary trunk is a rare congenital anomaly that accounts for $0.5 \%$ of congenital cardiac malformations. Mortality during infancy is $85 \%$ and survival into late adulthood is uncommon. The patient we describe may be the oldest survivor to be reported at the time of diagnosis. The main presenting feature was mitral regurgitation.

Cardiology

Department,

Birmingham

Heartlands Hospital

NHS Trust,

Birmingham

B T Saeed

R G Murray

Cardiothoracic

Surgery Department, Walsgrave Hospital

NHS Trust, Coventry

M D Rosin

Correspondence to

Dr Basil T Saeed,

Cardiology Department,

Nottingham City Hospital

NHS Trust, Hucknall Road,

Nottingham NG5 1PB.

\section{Case report} history of progressive dyspnoea and angina. Though chest pain and a systolic murmur had been noted in childhood, she remained well but with some physical limitation until the age of 50 when she sustained a lateral non- $Q$ wave myocardial infarction and continued to have post-infarction angina. At the age of 52 she was noted to be in atrial fibrillation and was treated with digoxin, diuretics, and $\beta$ blockers. At the age of 64 she presented with worsening angina and dyspnoea with
A 64-year-old white woman presented with a orthopnoea and paroxysmal nocturnal dyspnoea. There was biventricular hypertrophy with a harsh pansystolic murmur at the apex radiating to the axilla consistent with mitral regurgitation. The chest radiograph showed cardiomegaly with both left atrial and left ventricular enlargement and prominence of the pulmonary artery vasculature consistent with pulmonary hypertension. The electrocardiogram showed atrial fibrillation, left axis deviation, and partial left bundle branch block with a pattern typical of left ventricular hypertrophy and strain. Echocardiography and Doppler examination showed a dilated left ventricle and left atrium with end diastolic dimensions of $6.84 \mathrm{~mm}$ and $7.48 \mathrm{~mm}$ respectively. There was left ventricular hypertrophy with mild left ventricular dysfunction. Brightness of the chordae tendineae and the papillary muscle indicated dense nodular calcification, which also affected the posterior left ventricular wall. There was moderately severe mitral regurgitation.

Cardiac catheterisation was performed (table). A left to right shunt of $1 \cdot 5: 1$ was detected at the level of the main pulmonary artery. The cardiac index was $3.6 \mathrm{l} / \mathrm{min} / \mathrm{m}^{2}$ and left ventriculography showed left ventricular dilatation with moderate mitral regurgitation. The left coronary artery could not be engaged but the right coronary artery was enormously dilated, tortuous, and crossfilled the left coronary artery to its origin from the pulmonary artery. The left coronary artery was further filled via collaterals from a separate, small artery arising from the right coronary sinus (fig 1A). Contrast dye was noted in the main pulmonary artery. It entered through an anomalous sinus connection with the left coronary artery (fig 1B).

Surgical treatment was performed through a median sternotomy. The left atrium and

Cardiac catheterisation data

\begin{tabular}{lll}
\hline Site $\quad$ Pressure $(\mathrm{mm} \mathrm{Hg})$ & Oxygen saturation (\%) \\
\hline Inferior vena cava & - & 78 \\
Superior vena cava & - & 74 \\
Right atrium & $5^{\star}$ & 76 \\
Right ventricle & $40 / 5$ & 74.5 \\
Pulmonary artery & $32 / 15$ & $\mathbf{8 2}$ \\
Left ventricle & $130 / 15$ & $\overline{97}$ \\
Aorta & $140 / 80$ & $\mathbf{9 7}$ \\
\hline
\end{tabular}

^Mean value. 

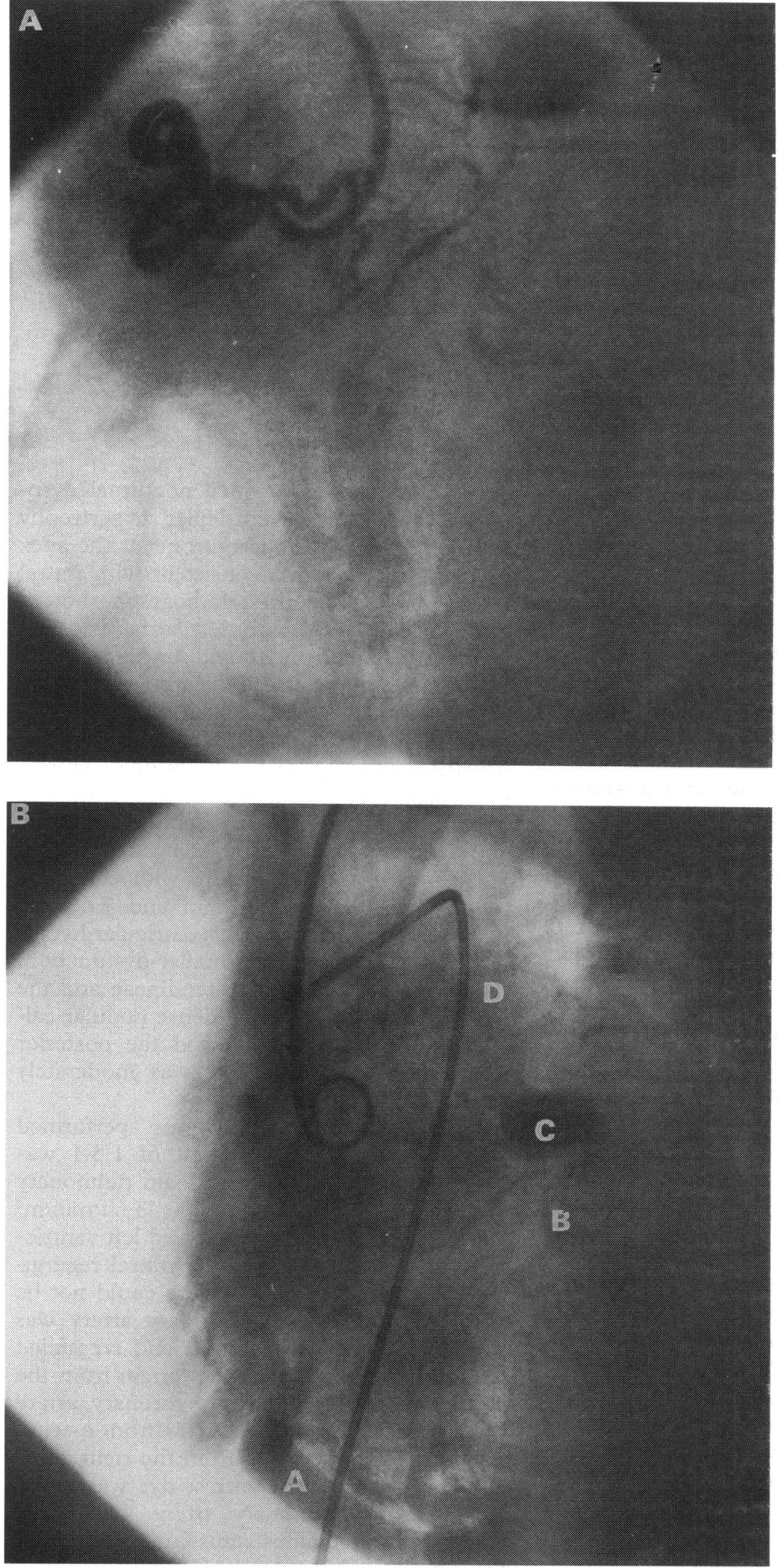

(A) A small and separate artery supplies collaterals to the left coronary artery. (B) Root aortogram obtained with a pigtail catheter. The tortuous and large right coronary artery $(A)$ crossfills the lefi coronary artery $(B)$. Contrast dye is shunting to the main pulmonary artery $(D)$ through a sinus connection $(C)$.

right ventricle were grossly dilated. There was a palpable thrill over the pulmonary artery. The right coronary artery was very dilated with a large left coronary artery arising from the posterior aspect of the pulmonary artery with its origin in the form of a dilated sinus. As noted from angiography, a feeding vessel to the left anterior descending coronary artery originated from the right coronary ostium and passed in front of the aorta. The left atrium was opened. The mitral valve was thickened and there was fusion of the median commissure but most of the abnormality was subvalvar, with gross calcification of the posteromedial papillary muscle and its attached chordae. The papillary muscle had been turned into a solid bar of calcium. The valve was excised and replaced with a $33 \mathrm{~mm}$ Sorin prosthesis. The pulmonary artery was opened longitudinally just above the valve and the origin of the left coronary was obliterated with a continuous 4.0 prolene suture. Because the left coronary artery and the left ventricle seemed to be well perfused by collateral circulation we decided not to bypass the vessel with a graft. The patient's hospital course was uncomplicated and she was well 2 years after operation.

\section{Discussion}

We believe that this patient may be the oldest survivor with anomalous origin of the left coronary artery from the pulmonary artery (Bland-White-Garland syndrome). Purut et al described only seven patients who were over the age of 50 years at the time of diagnosis: the oldest was 61 years. ${ }^{12}$

Only $10 \%$ of patients with this syndrome reach adulthood. Up to $50 \%$ of patients die suddenly, even those who are symptom free ${ }^{3}$; hence early diagnosis and correction of the anomaly is essential. As in this patient, both early onset angina, especially in childhood, and the presence of mitral regurgitation murmur suggest the diagnosis. Cross sectional echocardiography usually shows bright echoes from the papillary muscles, presumably reflecting ischaemic damage, ${ }^{4}$ but dense calcification of these structures and of the posterior left ventricular wall is a typical finding in longstanding cases. Echocardiography may also be useful in identifying the dilated coronary arteries. ${ }^{5}$ Doppler echocardiography and colour Doppler flow mapping were successful in detecting the abnormality and the site of the abnormal flow pattern from the left coronary artery into the pulmonary artery in diastole. ${ }^{4}$ The diagnosis is usually made from aortography or right coronary angiography or both. However, if not suspected, the anomaly could be missed even after invasive testing. ${ }^{4}$

There are three approaches to surgical treatment with none having a clear advantage over the others. The options are reimplantation, ligation, or ligation with saphenous vein bypass grafting (SVG). ${ }^{3}$ Evidence that the size of the right coronary artery became normal with involution of the collaterals in patients who had ligation with SVG suggested that simple ligation was an adequate treatment. This is supported by the fact that the main determinant of myocardial ischaemia was the presence of coronary steal with left to right shunting from the left coronary artery to the pulmonary artery and by the absence of ischaemia postoperatively despite graft occlu- 
sion in patients who had ligation with SVG. Previous reports also indicated that patients who had ligation without SVG did not have postoperative evidence of ischaemia. ${ }^{3}$ This was true of our patient whose symptoms improved significantly immediately after operation and in whom exercise thallium scintigraphy 8 months after operation showed no evidence of reverstble myocardial ischaemia in the left coronary artery territory.

Though surgical intervention was of great benefit in this elderly patient who had late complications, earlier diagnosis and correction of this anomaly might reduce the risk of high morbidity, which is mainly related to complications such as those we found in our patient-namely ischaemia with possible myocardial infarction, arrhythmia, and heart failure. Early surgery might also have avoided the need for mitral valve replacement: mitral regurgitation was reported to be reversible in young patients who had the anomaly corrected. ${ }^{6}$

We thank Mrs Tracey Ingram for typing the paper.

1 Purut CM, Sabiston DC. Origin of the left coronary artery from the pulmonary artery in older adults. If Thorac Cardiovasc Surg 1991;102:566-70.

2 Letcher JR, McCormick D, Tendler S, Ross J, Chandrasekaran $\mathrm{K}$, Brokman S. Left main coronary artery arising from the pulmonary trunk in a 56-year old patient presenting with acute myocardial infarction. $A m$ fCardiol 1991;68:1257-8.

3 Moodie DA, Fyfe D, Gill CC, et al. Anomalous origin of the left coronary artery from the pulmonary artery (Bland-White-Grarland syndrome) in adult patients: Long term follow up after surgery. Am Heart $f 1983$; 106:381-8.

4 Houston AB, Pollock JCS, Doig WB, et al. Anomalous origin of the left coronary artery from the pulmonary origin of the left coronary artery from the pulmonary
trunk: elucidation with colour Doppler flow mapping. trunk: elucidation with colo

5 Yoshikawa J, Katao H, Yanagihara K, et al. Non-invasive visualisation of the dilated main coronary arteries in coronary artery fistulas by cross-sectional echocardiography. Circulation 1982;65:600-3.

6 Ortiz De Salazar A, Juanena C, Aramendi JI, Castellano E, Cabrera A, Agosti J. Anomalous origin of the left coronary artery from the pulmonary artery. Surgical alternatives depending on the age of the patient. $f$ Cardiovasc Surg 1990;31:801-4. 